\title{
Hazard Regression Models of Early Mortality in Trauma Centers
}

\author{
David E Clark, MD, MPH, FACS, Jing Qian, PhD, Robert J Winchell, MD, and Rebecca A \\ Betensky, PhD \\ Maine Medical Center, Department of Surgery, Portland, ME (DEC, RJW) \\ Harvard School of Public Health, Harvard Injury Control Research Center, Cambridge, MA (DEC) \\ Harvard School of Public Health, Department of Biostatistics, Cambridge, MA (JQ, RAB)
}

\begin{abstract}
Background-Factors affecting early hospital deaths after trauma may be different from factors affecting later hospital deaths, and the distribution of short and long prehospital times may vary among hospitals. Hazard regression (HR) models may therefore be more useful than logistic regression (LR) models for analysis of trauma mortality, especially when treatment effects at different time points are of interest.
\end{abstract}

Study Design-We obtained data for trauma center patients from the 2008-9 National Trauma Data Bank (NTDB). Cases were included if they had complete data for prehospital times, hospital times, survival outcome, age, vital signs, and severity scores. Cases were excluded if pulseless on admission, transferred in or out, or ISS $<9$. Using covariates proposed for the Trauma Quality Improvement Program and an indicator for each hospital, we compared LR models predicting survival at 8 hours after injury to HR models with survival censored at 8 hours. HR models were then modified to allow time-varying hospital effects.

Results-85,327 patients in 161 hospitals met inclusion criteria. Crude hazards peaked initially, then steadily declined. When hazard ratios were assumed constant in HR models, they were similar to odds ratios in LR models associating increased mortality with increased age, firearm mechanism, increased severity, more deranged physiology, and estimated hospital-specific effects. However, when hospital effects were allowed to vary by time, HR models demonstrated that hospital outliers were not the same at different times after injury.

Conclusions-HR models with time-varying hazard ratios reveal inconsistencies in treatment effects, data quality, and/or timing of early death among trauma centers. HR models are generally more flexible than LR models, can be adapted for censored data, and potentially offer a better tool for analysis of factors affecting early death after injury.

\section{INTRODUCTION}

Most of the deaths due to serious injuries occur in the first few minutes after the traumatic event. $(1,2)$ For patients who survive until contact with the emergency medical services

\footnotetext{
C 2012 American College of Surgeons. Published by Elsevier Inc. All rights reserved.

Correspondence address: David E. Clark, MD, 887 Congress Street, Portland ME 04102, clarkd@ mmc.org; Telephone 207-774-2381; Fax 207-774-0459.

Publisher's Disclaimer: This is a PDF file of an unedited manuscript that has been accepted for publication. As a service to our customers we are providing this early version of the manuscript. The manuscript will undergo copyediting, typesetting, and review of the resulting proof before it is published in its final citable form. Please note that during the production process errors may be discovered which could affect the content, and all legal disclaimers that apply to the journal pertain.

Disclosure Information: Nothing to disclose.
} 
(EMS) system, prehospital and hospital care may reduce mortality, and differences in the quality of that care may produce measurable differences in how much mortality is reduced. However, interventions may have complex and potentially time-variant effects on the relatively crude outcome measures commonly analyzed. For example, if arrival at a hospital is delayed, fewer patients will survive until admission, but those that do arrive will be less likely to die subsequently, so differences in the quality of hospital care may have a lesser effect on the total number of deaths. Attempts to evaluate differences in outcome among trauma centers and trauma systems, especially those restricted to hospital registry data, should properly take into account such differences in the timing of hospital intervention.

Hazard regression (HR) models are a logical way to evaluate an outcome whose occurrence at a specific time is of interest. HR models are most familiar to clinicians involved in the care of cancer or other conditions where survival is measured in months or years, but the same mathematics can be applied to conditions where survival is measured in minutes or hours. In either case, HR models can be adapted to situations where patients are observed only during a portion of the time span, or where the effects of different covariates change over time. For acute injuries, the instantaneous risk of death is most strongly affected by time from the trauma event (so it makes sense to base a HR model on this time scale) but a hospital cannot affect the risk until the patient has been received alive (so it makes sense for the HR model to incorporate hospital effects only after this point, and not necessarily assume they are constant).

Several authors have suggested that trauma deaths occurring early in the course of hospital care should be considered separately from trauma deaths occurring later,(3-5) and it is certainly possible that the quality of care at a given hospital might vary, for example, between the emergency room and the operating room. It would be possible to construct separate logistic regression (LR) models for early and late deaths, but this might be cumbersome and would still not allow for variable periods of observation, interhospital transfers, and other types of censored data. On the other hand, HR models allow the effects of covariates (including individual hospitals) to vary over time in a flexible way that can incorporate as many different time periods as might be of interest. By extension, the same principles can be applied to care in the prehospital phase, if sufficient data on prehospital care become available.

The purpose of this study was to consider the use of HR models as a method for evaluating the early care of injured patients, to confirm that results from a simple HR model are essentially equivalent to those obtained from a LR model, and to demonstrate how additional insights can be obtained from modifications of HR models that cannot be obtained from LR models.

\section{METHODS}

\section{Data acquisition and inclusion criteria}

Data on patients admitted to US trauma centers in 2008 or 2009 were obtained from the National Trauma Data Bank (NTDB) Research Data Set, in compliance with its standard Data Use Agreement. An Institutional Review Board at Maine Medical Center judged the study to be exempt from review because it used only existing anonymous data. NTDB was established by the American College of Surgeons (ACS) in 1994, and collects trauma registry data from hundreds of hospitals across the US. For the first time, the 2008 NTDB data included the number of minutes between EMS activation and hospital arrival (prehospital time, abbreviated $t_{p}$ ), and the number of minutes between hospital arrival and death or other disposition (hospital time, abbreviated $t_{h}$ ). 
NTDB data files were converted for use with Stata (Version 11, StataCorp, College Station TX), and merged into a single analytic file. An outcome variable "died" was created if the patient either had an Emergency Room (ER) outcome of "Dead" or a hospital outcome of "Expired". Injury Severity Score (ISS), maximum Abbreviated Injury Score for the head or abdominal regions (AIShead, AISabd), Glasgow Coma Scale motor score (GCSm), systolic blood pressure (BP), pulse rate (PR), age, mechanism of injury, and status as a transfer from another hospital were categorized as described for predictive modeling by the ACS Trauma Quality Improvement Program (TQIP).(6)

We excluded all data from hospitals that were not ACS-verified Level I or Level II trauma centers, hospitals where more than $1 \%$ of cases were missing vital status (dead/alive) at discharge, or hospitals with fewer than 200 cases recording an Injury Severity Score (ISS) (7) of at least 9. Individual cases were then excluded if they had an ISS that was less than 9 or invalid, if they were declared "dead on arrival" (DOA), if they had no pulse at the time of hospital admission, or if they were transferred out to another institution.

In order to enable consistent statistical analyses, remaining cases were excluded if they had been managed first in another hospital and then transferred to the trauma center, if prehospital time $\left(t_{p}\right)$ could not be determined, or if the time until death $\left(t_{p}+t_{h}\right)$ could not be determined for patients who died. If a hospital had fewer than 100 cases after this exclusion, all of its cases were excluded. Cases excluded only because of missing time data were compared to those that had not been excluded for this reason.

\section{Brief statistical background}

When an outcome of interest can be expressed as the time until an event occurs, "time-toevent" statistical methods are the standard analytic approach. Because the most common applications of these statistical methods anticipate the occurrence of an adverse event (e.g., death), this approach is often referred to as "survival analysis". The outcome may be expressed as $S(t)$, the probability that a subject will survive at least until time $t$.

A related concept is the "hazard" function $h(t)$, which is the instantaneous probability at any given time that a subject (in our case, a patient) will experience the event in question at time $t$, given that they have not yet experienced the event. If we define an "integrated hazard" function

$$
H(t)=\int_{0}^{t} h(u) d u
$$

it can be shown that $H(t)=-\log (S(t))$.

Hazard regression (HR) models consider $h(t)$ to consist of some baseline hazard function, which may be expressed as $h_{0}(t)$, multiplied at any time $t$ by a factor that depends on individual characteristics $\left(z_{1}, z_{2}, \ldots z_{n}\right)$. Many applications are less concerned about the baseline hazard than the "hazard ratios" (or "incidence rate ratios") which measure the impact of the various characteristics and whether they increase or decrease $h(t)$.

A semiparametric (Cox) HR model makes no assumption about the form of the baseline hazard function, but assumes (subject to verification) that

$$
\operatorname{logh}(t)=\log h_{0}(t)+b_{1} z_{1}+b_{2} z_{2}+\ldots+b_{n} z_{n}
$$


Exponentiating both sides of the above equation gives

$$
h(t)=\left(h_{0}(t)\right)\left(e^{b_{1} z_{1}}\right)\left(e^{b_{2} z_{2}}\right) \ldots\left(e^{b_{n} z_{n}}\right) .
$$

Thus, the factors $\left(z_{1}, z_{2}, \ldots z_{n}\right)$ have multiplicative or proportional effects upon the baseline hazard. One of the factors in a HR (or LR) model may indicate a specific hospital (1 if the patient was treated in this hospital and 0 otherwise); the coefficient associated with this indicator variable then measures the effect of that hospital, adjusted for the other patientlevel variables.

When there is "left truncation" (in our case, deaths before hospital arrival), HR models can be constructed so that patients enter into the calculations only for the times during which they are actually under observation. The factors for a given patient and/or hospital $\left(z_{1}, z_{2}, \ldots\right.$ $\left.z_{n}\right)$ may also be allowed to vary at different times, and their effects $\left(b_{1}, b_{2}, \ldots b_{n}\right)$ may also be modeled as constant or time-varying. The assumption of proportional hazards can be verified by obtaining a Kaplan-Meier estimate of $S(t)$, calculating $H(t)$ from the relationship shown above, and plotting $H(t)$ for groups defined by the covariate of interest; under proportionality, these should be approximately parallel. If there are serious deviations from proportionality, the model can be broken up "piecewise" to reflect different effects in different time periods. These considerations allow great flexibility in the application of HR models.

\section{Analysis for this study}

An outcome of "early death" was arbitrarily defined as death occurring within a total elapsed time $\left(t_{p}+t_{h}\right)$ no greater than 8 hours (480 minutes) after the injury event. Preliminary analyses also evaluated "early death" thresholds of 6 hours or 12 hours. Patients who died after the threshold time were considered "survivors" in the "early death" models. Since the data do not include any information about persons who were not brought to a hospital with detectable vital signs, those who are included consititute a left truncated sample of all trauma deaths, restricted to those who have survived long enough to reach a hospital. The Stata procedure "stset" allowed this structure to be incorporated in the HR models by designating the entry time as $t_{p}$ and designating the exit time as the minimum of the time from injury until death or 480 minutes.

For included patients, a logistic regression (LR) model was first constructed as proposed for the ACS Trauma Quality Improvement Program (TQIP),(6) but using "early death" as an endpoint. In addition to the TQIP factors, the LR model included vital signs measured prior to hospital arrival (in addition to those measured at the time of arrival), prehospital time, and indicator variables designating each hospital. In order to maintain comparability with TQIP, we did not make any further modifications to variables or interactions. Using the same covariates included in the LR model, a HR model was constructed, censoring all observations at 8 hours.

For each hospital, LR and HR models were then constructed with an indicator variable for each index hospital. The coefficients estimated for the indicator variables in these models (relative to the other hospitals as a reference group) were interpreted as a log odds ratio and a log hazard ratio measuring the hospital effect on mortality, assuming that the effect was constant for each hospital. For each hospital, HR models were also constructed in which the hospital-specific hazards were estimated separately (piecewise) for the time periods 0-60 minutes, 61-120 minutes, and 121-480 minutes following the presumed time of injury. Each time-specific and hospital-specific hazard estimate was compared to the overall hospital- 
specific estimate as well as to the time-specific estimate (relative to the other hospitals as a reference group).

After completing the above analyses, the process was repeated including patients who had been transferred from another hospital. A variable indicating the transfer status of the patient ( 1 if the patient had been transferred and 0 otherwise) was added to the models as a covariate. For the transferred cases, $t_{p}$ included time spent at the referring hospital, and entry time was specified as $t_{p}$ in these models as it had been for the models that excluded transferred patients. In order to distinguish the effects of prehospital time with or without the involvement of a previous hospital, the interactions of categorized prehospital time and transfer status were also added as indicator variables in the models that included transferred patients. Results were then compared to the analyses that had excluded interhospital transfers.

\section{RESULTS}

The NTDB contained 226,734 records of patients admitted during 2008-2009 to an ACSverified Level I-II trauma center, excluding institutions that had fewer than 200 patients with ISS of at least 9 , or more than $20 \%$ with missing data. Of these 226,734 cases, only 85,327 $(37.6 \%)$ were direct admissions that also had valid data for prehospital time, as well as time of death for patients who died.

Characteristics of the patients included in the principal analysis are summarized in Table 1. Among the excluded cases were 65,741 (30\%) who had been transferred in from another hospital. Of the remaining 75,666 cases excluded for missing time data, there were 9,515 deaths $(12.6 \%)$ compared to 3,019 deaths $(3.5 \%)$ in the cases where time data were recorded. Patients excluded for missing time data were somewhat more likely to be older than 65 years ( $25.5 \%$ vs. $20.6 \%)$, less likely to be injured by firearm ( $7.6 \%$ vs. $8.0 \%)$, more likely to have a GCSm of 1 on admission (11.5\% vs. 8.4\%), but less likely to have a GCSm of $2-5$ on admission $(5.7 \%$ vs. $7.0 \%)$.

Patients selected for analysis were distributed among 161 hospitals, with case volumes ranging from 111 to 1671 patients. $85(53 \%)$ of these hospitals were trauma centers with an ACS verification as Level I, 74 (46\%) had an ACS verification as Level II, and 2 changed levels from one year to the next. The percentage of patients arriving at a hospital within 60 minutes of injury was $79 \%$ and ranged from $39 \%$ to $99 \%$ for different hospitals.

Calculation of an overall crude hazard rate, estimated at 15-minute intervals and not adjusted for covariates, is shown in Table 2. The hazard rate had an initial peak and gradually decreased over the 8 hour period of observation. Slight fluctuations in the numbers of deaths for successive time periods did not appear to constitute a trimodal or other anomalous distribution. Analyses using a threshold of 6 hours or 12 hours to define "early death" were very similar to those using an 8 hour threshold.

Table 3 shows the odds ratios for patient-level covariates obtained from multiple logistic regression with the outcome of death within 8 hours of injury, compared with the hazard ratios for the same covariates in a HR model censored at 8 hours after injury (assuming a constant effect of the covariates over the 8 hours).

When hospital effects were considered constant, the distribution of these effects measured using hazard ratios was very similar to the distribution using odds ratios obtained from a LR model, as depicted in Figure 1 (in which the log odds ratio and the log hazard ratio are divided by their standard errors to make them comparable). However, when hospital effects were allowed to vary among the time periods 0-60 minutes, 61-120 minutes, and 121-480 
minutes, there were considerable differences in the apparent effects at different times, although the absolute numbers of deaths were relatively small and the observed differences generally did not reach statistical significance.

Table 4 shows odds ratios and hazard ratios where a 99\% confidence interval of the hospital effect in the LR model or any of the HR models did not include the null value. There were many more high-mortality outliers than low-mortality outliers. The hazard ratio estimating the hospital effect specifically for the first hour or two was often quite different from that estimated with the assumption of a constant hazard ratio over eight hours. For example, Hospital B in Table 4 had a very high mortality for patients seen during the first hour after injury yet would not have been considered an outlier by conventional LR analysis.

Only 122 patients had been excluded because they had been transferred out of the index hospital within the first 8 hours. However, 21,022 patients had been initially excluded because they had been transferred from another hospital to the index hospital. Analyses were repeated with the latter group included. The effects of transfer status, or the interaction of transfer status and categorized prehospital time, were not significant in the overall LR or HR models. Individual hospital effects with and without transfers were very similar (Figure 2).

Of the 13 hospitals shown as outliers in Table 4 (with an arbitrary cutoff of $p<.01$ ), 12 would also appear in a similar table if transfers were included, and 2 others would be added.

\section{DISCUSSION}

Most studies of patients hospitalized after serious injury simply consider the outcome of survival at the time of hospital discharge, given that the patient was alive at the time of admission. Although very sophisticated and complicated mathematical models have been constructed to predict this outcome, $(8-10)$ their validity is limited by their failure to incorporate information about the time periods before or after hospital observation.

Previous investigations have demonstrated that it may be inappropriate to consider hospital mortality, length of stay, and discharge destination independently.(5) A difference in the probability of survival at the time of hospital discharge does not necessarily imply that a similar difference will be found at the end of a specified time period.(11) Ideally, the patient's condition at a fixed follow-up time of 30 days or more after injury would be a preferable binary outcome to measure or predict,(12) but appropriately constructed HR or other models may also be able to account for some of the differences in discharge timing and destination. $(13,14)$

Arrival times may also vary from one hospital to another, especially between urban areas with rapid EMS transport times and rural areas where prolonged transportation times may result in fewer patients reaching a hospital alive. Models that do not properly allow for the "left truncated" population (that is, persons who are not transported to a hospital because they have already died) may lead to incorrect conclusions about the effect of prehospital delay.(15) HR models may also be useful in evaluating this early mortality, because they can explicitly identify the times when specific patients are actually observed in a given hospital and account for the differences in underlying risk associated with a specific time after an injury has occurred. The technique also potentially allows for a similar analysis of mortality in the prehospital phase, should such data be available, and hence provides an approach that can be extended to a more comprehensive system-based model.

Patients received in transfer from another hospital may be difficult to compare with patients received directly from the scene of an injury.(16) On the one hand, the reason for transfer is often that their injuries are more serious; on the other hand, their survival long enough to reach a second hospital implies some degree of physiologic stability. In order to focus on a 
uniform population, our main analysis excluded patients received in transfer. However, interhospital differences in early mortality were not much different when transfer patients were included.

Patients transferred out to another hospital also complicate any analysis of mortality. If a given hospital sends away its most serious cases, its measured effect on mortality will be falsely low. Excluding these cases from either HR or LR analysis may also introduce bias when considering hospital effects on outcome; censoring these cases in HR models is still biased because the censoring is not independent from the outcome. Few patients are transferred acutely out of a major trauma center, so these considerations do not affect the analysis in the present study. However, for a dataset containing hospitals that frequently transfer patients out in the acute phase, a possible approach would be to determine the consistency of results from analyses that exclude these cases, include them as deaths, or censor them as survivors.

If a personal identifier were available in the data that allowed tracking an individual patient from one hospital to another, it might be possible to identify transfer patients as censored from one hospital and reappearing as a readmission in another hospital. HR models could then be constructed to allow for these situations, measuring the effect of each hospital only during the time period when the given patient was actually under the care of its staff, and adding a time-dependent covariate to indicate transfer status.

Whether a LR or a HR model has been used to estimate hospital effects, the imprecision of these effect estimates also needs to be considered. Typically, confidence limits are calculated from the model, and hospitals whose confidence limits do not include a null value (e.g., an odds ratio or hazard ratio of 1) are considered "outliers". However, testing multiple hospitals against a standard theoretically increases the frequency of Type I errors, and results may vary depending upon whether and how the confidence limits are adjusted to reduce this frequency. $(17,18)$ For public reporting or reimbursement decisions, some adjustment is probably warranted. The p-value threshold of $0.00031(.05 / 161)$ in Table 4 corresponds to a Bonferroni correction of the p-value threshold of 0.05 when 161 hospital comparisons have been made.

Several authors have recently suggested that "multilevel" or "hierarchical" LR models may be preferable when comparing trauma center performance to a standard.(19-21) The potential advantages include labeling fewer hospitals as outliers, especially hospitals with smaller volumes of patients. HR models can implement a similar approach by treating the hospital effects as random rather than fixed, a modification referred to as "shared frailty". The random effects approach may reduce concerns about multiple testing, although this opinion is not held by all statisticians.(18)

Our findings in this study confirm that the mortality hazard for injured patients admitted to a hospital has an initial peak followed by an essentially monotonic decrease. No deaths were recorded among the few patients admitted less than 15 minutes after injury (Table 2), but there is an understandable delay in the declaration of death for the most acute cases while the diagnosis is confirmed and resuscitative measures are being carried out.(2) "Interval censoring" allows HR models to accommodate these situations when the time of death cannot be exactly specified. $(22,23)$

There was a general similarity of covariate effects in Table 3 between a LR model (using death within 8 hours as an outcome) and a HR model (censoring cases still alive at 8 hours). Theoretical reasons for this similarity are discussed elsewhere.(24-27) Increased age, firearm mechanism, increased severity, and more deranged physiology all were associated with increased mortality. Having controlled for ISS and GCSm, patients with serious head 
trauma were less likely to die within the initial 8-hour period of observation; this finding is consistent both with the physiology of death following traumatic brain injury and with the clinical practice of initial support and observation. Hospital mortality could be affected by differences in this clinical practice related to the time period of observation and local practices regarding early withdrawal of care in patients deemed to have a low probability of functional survival.

Increasing length of prehospital time was associated with lower mortality (among patients who reached the hospital alive) both in LR and HR models, a result that should be expected given that prehospital deaths have been truncated. Simply including prehospital time as a covariate in either type of model would control somewhat for patient acuity, but would require additional assumptions about independence of these times, other covariate effects, and hospital effects unless numerous interaction terms were added. HR models that include time-varying hospital effects as well as prehospital times improve model flexibility and interpretation. Furthermore, the ability to include similar time-varying prehospital effects also enhances the potential utility of HR models.

Hospital effects as shown in the first two columns of Table 4 were also relatively similar between a LR model (using only death within 8 hours as an outcome) and a HR model (censoring cases still alive at 8 hours). When hazard ratios were allowed to vary by time period, however, there were interesting differences. In particular, several of the hospitals reported no deaths within the first or even second hour. Although we had excluded patients declared dead on arrival and those with no pulse on arrival, these findings suggest that differences in measured outcomes may be due to differences in data quality as well as differences in clinical quality.

The collection of prehospital data in the NTDB is inherently challenging. There is no nationally accepted standard for EMS reporting to hospitals, EMS run sheets are not uniform, and reports are not universally available at the time of admission. Thus, the data must be gleaned from a collection of different EMS records by hospital-based personnel, and the frequency with which these data points are collected and their accuracy is a recognized weakness within the NTDB. Some of these problems have great potential to be improved with the current development of a National EMS Information System (NEMSIS).

Our study is further limited by the lack of data on prehospital time and time to death in a substantial number of NTDB records. This could be remedied to some degree by imputation methods,(28) but seems to be more frequent in fatal cases and could affect conclusions about outcomes, as could any other missing or miscoded data used for risk adjustment. However, these limitations should become less of a problem as the recording of prehospital and hospital times to the minute becomes more standard for trauma registries and data quality generally improves as a result of the TQIP program.

We believe our findings show that HR models have promise in the evaluation of hospital mortality after injury within a system of care that includes both prehospital and hospital factors, and where the period of observation and nature of interventions may vary during different phases of care. By extension, these techniques are also applicable to the analysis of care after myocardial infarction, stroke, or other acute conditions in which outcome is dependent upon a time-limited, system-based approach.

\section{Acknowledgments}

Supported by Grant R01CE001594 from the National Center for Injury Prevention and Control. 


\section{REFERENCES}

1. Acosta JA, Yang JC, Winchell RJ, et al. Lethal injuries and time to death in a level I trauma center. J Am Coll Surg. 1998; 186:528-533. [PubMed: 9583692]

2. Clark DE, Qian J, Sihler KC, et al. The distribution of survival times after injury. World J Surg. 2012; 36:1562-1570. [PubMed: 22402976]

3. Osler T. Injury severity scoring: Perspectives in development and future directions. Am J Surg. 1993; 165:43S-51S. [PubMed: 8438999]

4. Mullins RJ, Mann NC, Brand DM, Lenfesty BS. Specifications for calculation of risk-adjusted odds of death using trauma registry data. Am J Surg. 1997; 173:422-425. [PubMed: 9168081]

5. Clark DE, Lucas FL, Ryan LM. Predicting hospital mortality, length of stay, and transfer to longterm care for injured patients. J Trauma. 2007; 62:592-600. [PubMed: 17414333]

6. Hemmila MR, Nathens AB, Shafi S, et al. The Trauma Quality Improvement Program: Pilot study and initial demonstration of feasibility. J Trauma. 2010; 68:253-262. [PubMed: 20154535]

7. Baker SP, O'Neill B, Haddon W Jr, Long WB. The injury severity score: A method for describing patients with multiple injuries and evaluating emergency care. J Trauma. 1974; 14:187-196. [PubMed: 4814394]

8. Burd RS, Ouyang M, Madigan D. Bayesian logistic injury severity score: A method for predicting mortality using International Classification of Disease-9 codes. Acad Emerg Med. 2008; 15:466475. [PubMed: 18439203]

9. Glance LG, Osler TM, Mukamel DB, et al. TMPM-ICD9: A trauma mortality prediction model based on ICD-9-CM codes. Ann Surg. 2009; 249:1032-1039. [PubMed: 19474696]

10. Moore L, Hanley JA, Turgeon AF, et al. A new method for evaluating trauma centre outcome performance: TRAM-adjusted mortality estimates. Ann Surg. 2010; 251:952-958. [PubMed: 20395844]

11. Gorra AS, Clark DE, Mullins RJ, Delorenzo MA. Regional variation in hospital mortality and 30day mortality for injured Medicare patients. World J Surg. 2008; 32:954-959. [PubMed: 18224464]

12. Hutter MM, Rowell KS, Devaney LA, et al. Identification of surgical complications and deaths: An assessment of the traditional surgical morbidity and mortality conference compared with the American College of Surgeons - National Surgical Quality Improvement Program. J Am Coll Surg. 2006; 203:618-624. [PubMed: 17084322]

13. Clark DE, Ryan LM, Lucas FL. A multi-state piecewise exponential model of hospital outcomes after injury. J Appl Statistics. 2007; 34:1225-1239.

14. Gorra AS, Clark DE, Mullins RJ. Using hospital outcomes to predict 30-day mortality among injured patients insured by Medicare. Arch Surg. 2011; 146:195-200. [PubMed: 21339432]

15. Newgard CD, Schmicker RH, Hedges JR, et al. Emergency medical services intervals and survival in trauma: assessment of the "golden hour" in a North American prospective cohort. Ann Emerg Med. 2010; 55:235-246. [PubMed: 19783323]

16. Rivara FP, Koepsell TD, Wang J, et al. Outcomes of trauma patients after transfer to a level I trauma center. J Trauma. 2008; 64:1594-1599. [PubMed: 18545129]

17. Bilimoria KY, Cohen ME, Merkow RP, et al. Comparison of outlier identification methods in hospital surgical quality improvement programs. J Gastrointest Surg. 2010; 14:1600-1607. [PubMed: 20824379]

18. Jones HE, Ohlssen DI, Spiegelhalter DJ. Use of the false discovery rate when comparing multiple health care providers. J Clin Epidemiol. 2008; 61:232-240. [PubMed: 18226745]

19. Clark DE, Hannan EL, Wu C. Predicting risk-adjusted mortality for trauma patients: Logistic versus multilevel logistic models. J Am Coll Surg. 2010; 211:224-231. [PubMed: 20670860]

20. Moore L, Hanley JA, Turgeon AF, Lavoie A. Evaluating the performance of trauma centers: Hierarchical modeling should be used. J Trauma. 2010; 69:1132-1137. [PubMed: 20404760]

21. Glance LG, Osler TM, Dick AW, et al. The Survival Measurement and Reporting Trial for Trauma (SMARTT): Background and study design. J Trauma. 2010; 68:1491-1497. [PubMed: 20068485]

22. Lindsey JC, Ryan LM. Tutorial in biostatistics: Methods for interval-censored data. Stat Med. 1998; 17:219-238. [PubMed: 9483730] 
23. Zhang Z, Sun J. Interval censoring. Stat Methods Med Res. 2010; 19:53-70. [PubMed: 19654168]

24. Ingram DD, Kleinman JC. Empirical comparisons of proportional hazards and logistic regression models. Stat Med. 1989; 8:525-538. [PubMed: 2727473]

25. Doksum KA, Gasko M. On a correspondence between models in binary regression analysis and in survival analysis. Int Stat Rev. 1990; 58:243-252.

26. D'Agostino RB, Lee ML, Belanger AJ, et al. Relation of pooled logistic regression to time dependent Cox regression analysis: the Framingham Heart Study. Stat Med. 1990; 9:1501-1515. [PubMed: 2281238]

27. Symons MJ, Moore DT. Hazard rate ratio and prospective epidemiological studies. J Clin Epidemiol. 2002; 55:893-899. [PubMed: 12393077]

28. Moore L, Hanley JA, Turgeon AF, et al. A multiple imputation model for imputing missing physiologic data in the National Trauma Data Bank. J Am Coll Surg. 2009; 209:572-579. [PubMed: 19854396] 


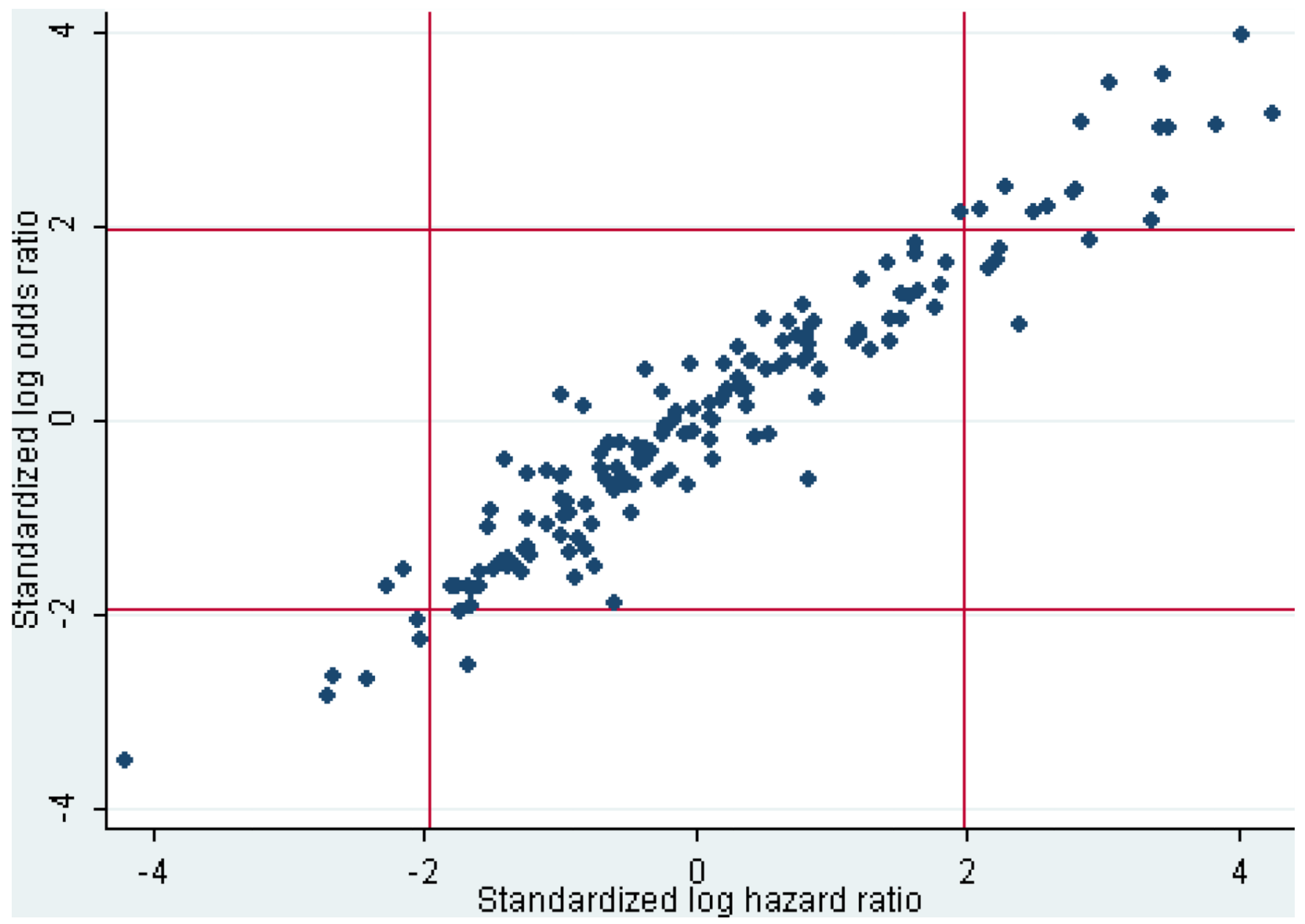

Figure 1.

Standardized log odds ratios compared with standardized log hazard ratios for the effect on mortality attributable to the 161 sampled hospitals. The distribution of hospital effects is very similar with either model $(\mathrm{r}=0.96)$. 


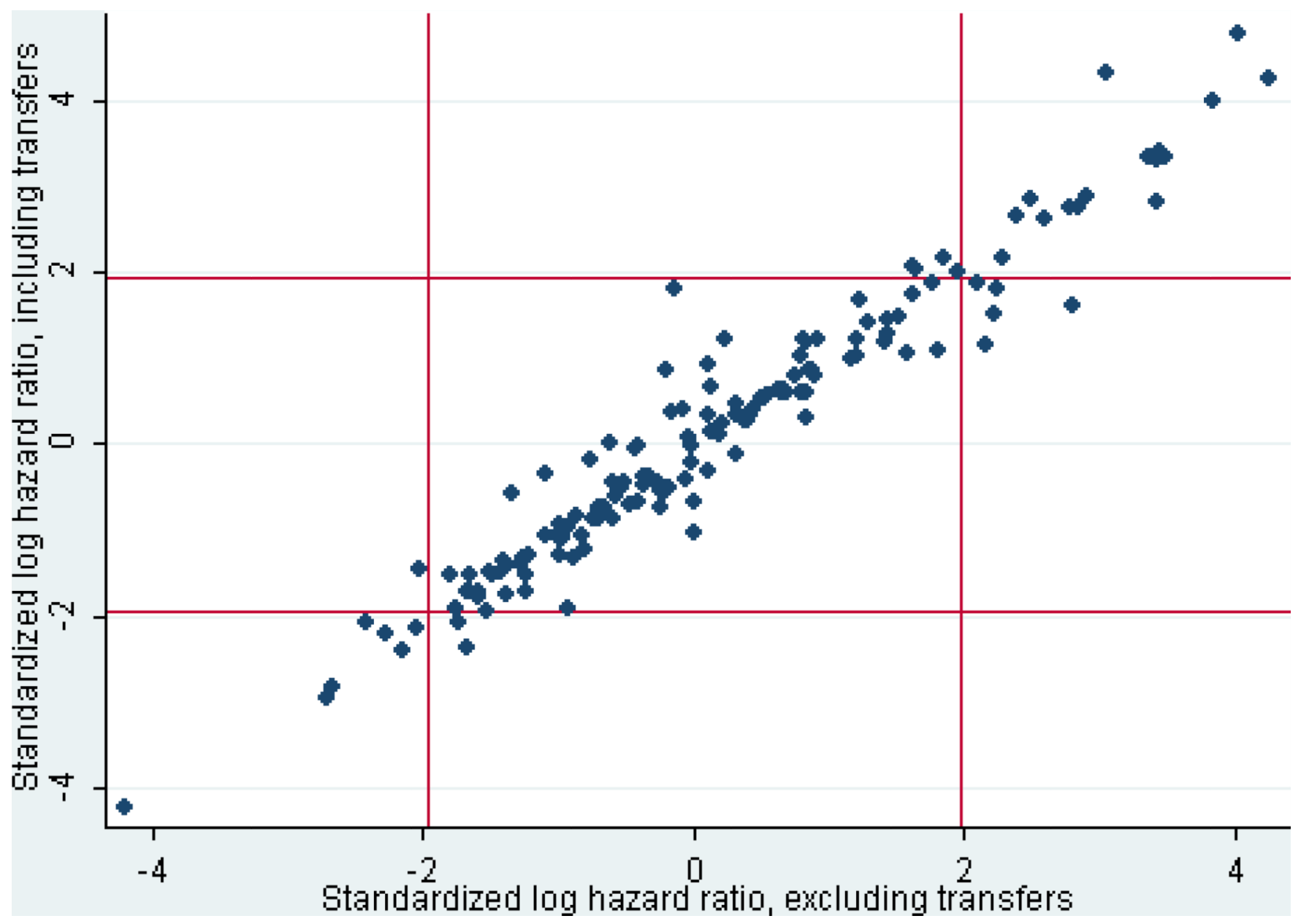

Figure 2.

Standardized log hazard ratios with transferred patients included, compared with standardized log hazard ratios with transferred patients excluded. The distribution of hospital effects is very similar with either model $(\mathrm{r}=0.97)$. 
Table 1

Patient Characteristics for the Analysis Sample, NTDB 2008-2009

\begin{tabular}{|c|c|c|c|}
\hline & $\begin{array}{l}\text { Died within } 8 \mathrm{~h} \\
(\mathrm{n}=1,943)\end{array}$ & $\begin{array}{l}\text { Survived } 8 \text { h } \\
(n=83,384)\end{array}$ & $\begin{array}{l}\text { Total } \\
(\mathbf{n}=85,327)\end{array}$ \\
\hline Died in hospital & 1,943 & $1,076(1.3 \%)$ & $3,019(3.5 \%)$ \\
\hline Died within $8 \mathrm{~h}$ & 1,943 & 0 & $1,943(2.3 \%)$ \\
\hline Age $>=65 y$ & $400(20.6 \%)$ & $17,215(20.6 \%)$ & $17,615(20.6 \%)$ \\
\hline Firearm injury & $622(32.0 \%)$ & $6,238(7.5 \%)$ & $6,860(8.0 \%)$ \\
\hline ISS 25-75 & $1,214(62.5 \%)$ & $10,516(12.6 \%)$ & $11,730(13.7 \%)$ \\
\hline \multicolumn{4}{|l|}{ Head AIScat } \\
\hline AIS 1-2 & $129(6.6 \%)$ & $12,589(15.1 \%)$ & $12,718(14.9 \%)$ \\
\hline AIS 3-4 & $436(22.4 \%)$ & $26,221(31.4 \%)$ & $26,657(31.2 \%)$ \\
\hline AIS 5-6 & $643(33.1 \%)$ & $2,253(2.7 \%)$ & $2,896(3.4 \%)$ \\
\hline \multicolumn{4}{|l|}{ Abdomen AIScat } \\
\hline AIS 1-2 & $372(19.1 \%)$ & $16,066(19.3 \%)$ & $16,438(19.3 \%)$ \\
\hline AIS 3-4 & $550(28.3 \%)$ & $6,469(7.8 \%)$ & $7,019(8.2 \%)$ \\
\hline AIS 5-6 & $72(3.7 \%)$ & $553(0.7 \%)$ & $625(0.7 \%)$ \\
\hline \multicolumn{4}{|l|}{ Prehospital times } \\
\hline $0-30 \mathrm{~min}$ & $757(39.0 \%)$ & $17,669(21.2 \%)$ & $18,426(21.6 \%)$ \\
\hline $31-45 \min$ & $626(32.2 \%)$ & $28,829(34.6 \%)$ & $29,455(34.5 \%)$ \\
\hline $46-60 \mathrm{~min}$ & $275(14.2 \%)$ & $19,366(23.2 \%)$ & $19,641(23.0 \%)$ \\
\hline $61-90 \mathrm{~min}$ & $227(11.7 \%)$ & $13,621(16.3 \%)$ & $13,848(16.2 \%)$ \\
\hline$>90 \mathrm{~min}$ & $58(3.0 \%)$ & $3,899(4.7 \%)$ & $3,957(4.6 \%)$ \\
\hline \multicolumn{4}{|c|}{ Prehospital physiology } \\
\hline Pulse $<=40$ & $153(7.9 \%)$ & $359(0.4 \%)$ & $512(0.6 \%)$ \\
\hline BP 0 & $247(12.7 \%)$ & $281(0.3 \%)$ & $528(0.6 \%)$ \\
\hline BP $1-89 \mathrm{mmHg}$ & $337(17.3 \%)$ & $3,446(4.1 \%)$ & $3,783(4.4 \%)$ \\
\hline GCSm 1 & $1,041(53.6 \%)$ & $3,805(4.6 \%)$ & $4,846(5.7 \%)$ \\
\hline GCSm 2-5 & $357(18.4 \%)$ & $8,218(9.9 \%)$ & $8,575(10.0 \%)$ \\
\hline \multicolumn{4}{|c|}{ Admission physiology } \\
\hline Pulse $<=40$ & $81(4.2 \%)$ & $228(0.3 \%)$ & $309(0.4 \%)$ \\
\hline BP 0 & $203(10.4 \%)$ & $95(0.1 \%)$ & $298(0.3 \%)$ \\
\hline BP $1-89 \mathrm{mmHg}$ & $576(29.6 \%)$ & $3,086(3.7 \%)$ & $3,662(4.3 \%)$ \\
\hline GCSm 1 & $1,355(69.7 \%)$ & $5,813(7.0 \%)$ & $7,168(8.4 \%)$ \\
\hline GCSm 2-5 & $234(12.0 \%)$ & $5,742(6.9 \%)$ & $5,976(7.0 \%)$ \\
\hline
\end{tabular}

ISS, Injury Severity Score (maximum 75); AIScat, Maximum Abbreviated Injury Score, categorized (maximum 6); BP, blood pressure (systolic); GCSm, Glasgow Coma Scale, motor component (minimum 1, normally 6). 
Table 2

Crude Hazards at 15-min Intervals after Injury, for Patients Who Have Arrived at a Hospital with Detectable Vital Signs

\begin{tabular}{|c|c|c|c|c|}
\hline $\begin{array}{l}\text { Time in minutes } \\
\left(t_{p}+t_{h}\right)\end{array}$ & $\begin{array}{l}\text { Patients at risk } \\
S\end{array}$ & $\begin{array}{l}\text { Admissions } \\
a\end{array}$ & $\begin{array}{l}\text { Deaths } \\
d\end{array}$ & $\begin{array}{l}\text { Hazard } \\
d / S\end{array}$ \\
\hline $0-14$ & 0 & 640 & 0 & - \\
\hline $15-29$ & 640 & 15,873 & 11 & 0.0172 \\
\hline $30-44$ & 16,502 & 29,594 & 77 & 0.0047 \\
\hline $45-59$ & 46,019 & 20,435 & 74 & 0.0016 \\
\hline $60-74$ & 66,380 & 10,100 & 87 & 0.0013 \\
\hline $75-89$ & 76,373 & 4,523 & 98 & 0.0013 \\
\hline $90-104$ & 80,818 & 2,069 & 122 & 0.0015 \\
\hline $105-119$ & 82,765 & 941 & 97 & 0.0012 \\
\hline $120-134$ & 83,609 & 383 & 86 & 0.0010 \\
\hline $135-149$ & 83,906 & 273 & 103 & 0.0012 \\
\hline $150-164$ & 84,076 & 124 & 75 & 0.0009 \\
\hline $165-179$ & 84,125 & 93 & 88 & 0.0010 \\
\hline $180-194$ & 84,130 & 60 & 86 & 0.0010 \\
\hline $195-209$ & 84,104 & 37 & 85 & 0.0010 \\
\hline $210-224$ & 84,056 & 37 & 81 & 0.0010 \\
\hline $225-239$ & 84,102 & 23 & 77 & 0.0009 \\
\hline $240-254$ & 83,958 & 19 & 76 & 0.0009 \\
\hline $255-269$ & 83,901 & 12 & 61 & 0.0007 \\
\hline $270-284$ & 83,852 & 13 & 56 & 0.0007 \\
\hline $285-299$ & 83,809 & 7 & 57 & 0.0007 \\
\hline $300-314$ & 83,759 & 9 & 41 & 0.0005 \\
\hline $315-329$ & 83,727 & 13 & 44 & 0.0005 \\
\hline $330-344$ & 83,696 & 10 & 48 & 0.0006 \\
\hline $345-359$ & 83,658 & 3 & 49 & 0.0006 \\
\hline $360-374$ & 83,612 & 9 & 46 & 0.0006 \\
\hline $375-389$ & 83,575 & 5 & 37 & 0.0004 \\
\hline $390-404$ & 85,327 & 3 & 31 & 0.0004 \\
\hline $405-419$ & 83,515 & 5 & 17 & 0.0002 \\
\hline $420-434$ & 83,503 & 5 & 32 & 0.0004 \\
\hline $435-449$ & 83,476 & 5 & 34 & 0.0004 \\
\hline $450-464$ & 83,447 & 2 & 32 & 0.0004 \\
\hline $465-479$ & 83,417 & 2 & 33 & 0.0004 \\
\hline
\end{tabular}

At the beginning or each time interval $i$, the number or patients at risk $S_{i}$ may be calculated as $S_{i-1}+a_{i-1}-d_{i-1}$. The hazard is the rate of death among the patients at risk during the specified time interval. Time after injury is calculated as prehospital time $\left(t_{p}\right)$ plus hospital time $\left(t_{h}\right)$. HR models described elsewhere in this article calculate the hazard for each minute rather than for 15-minute intervals. 


\section{Table 3}

Results of Multivariable Logistic Regression Model Predicting Early Death ( $<=8$ h after Injury) and Cox Proportional Hazard Regression Model (Censored At 8 h after Injury)

\begin{tabular}{|c|c|c|}
\hline & $\begin{array}{l}\text { Odds ratios } \\
\text { (with } 95 \% \text { CI) }\end{array}$ & $\begin{array}{l}\text { Hazard ratios } \\
\text { (with } 95 \% \mathrm{CI} \text { ) }\end{array}$ \\
\hline Age $>=65$ vs $<65$ years & $4.33(3.71-5.06)$ & $2.91(2.58-3.28)$ \\
\hline Firearm injury & $4.04(3.51-4.66)$ & $2.99(2.68-3.35)$ \\
\hline ISS $25-75$ vs $<25$ & $3.00(2.60-3.48)$ & $2.51(2.23-2.83)$ \\
\hline Head AIScat & $0.86(0.80-0.92)$ & $0.89(0.84-0.94)$ \\
\hline Abdomen AIScat & $1.70(1.57-1.83)$ & $1.46(1.38-1.55)$ \\
\hline \multicolumn{3}{|l|}{ Prehospital times } \\
\hline $1-30 \mathrm{~min}$ & 1.00 (referent) & 1.00 (referent) \\
\hline $31-45 \mathrm{~min}$ & $0.84(0.72-0.96)$ & $0.89(0.79-0.99)$ \\
\hline $46-60 \min$ & $0.56(0.47-0.68)$ & $0.63(0.54-0.73)$ \\
\hline $61-90 \mathrm{~min}$ & $0.41(0.34-0.51)$ & $0.53(0.45-0.63)$ \\
\hline$>90 \mathrm{~min}$ & $0.29(0.21-0.41)$ & $0.41(0.31-0.55)$ \\
\hline \multicolumn{3}{|l|}{ Prehospital physiology } \\
\hline Pulse $<=40$ vs $>40$ & $1.66(1.16-2.36)$ & $1.91(1.55-2.37)$ \\
\hline BP 0 vs $>=90 \mathrm{mmHg}$ & $3.19(2.37-4.29)$ & $1.47(1.21-1.77)$ \\
\hline BP $1-89$ vs $>=90 \mathrm{mmHg}$ & $1.55(1.31-1.83)$ & $1.41(1.24-1.61)$ \\
\hline GCSm 1 vs 6 & $2.81(2.33-3.39)$ & $2.18(1.87-2.55)$ \\
\hline GCSm $2-5$ vs 6 & $1.48(1.23-1.78)$ & $1.33(1.13-1.57)$ \\
\hline \multicolumn{3}{|l|}{ Admission physiology } \\
\hline Pulse $<=40$ vs $>40$ & $2.84(1.81-4.45)$ & $2.98(2.31-3.83)$ \\
\hline $\mathrm{BP} 0 \mathrm{vs}>=90 \mathrm{mmHg}$ & $13.84(9.81-19.5)$ & $7.05(5.81-8.54)$ \\
\hline BP $1-89$ vs $>=90 \mathrm{mmHg}$ & $3.60(3.13-4.14)$ & $2.76(2.47-3.09)$ \\
\hline GCSm 1 vs 6 & $16.71(13.7-20.4)$ & $14.52(12.2-17.3)$ \\
\hline GCSm $2-5$ vs 6 & $5.00(4.06-6.14)$ & $5.06(4.18-6.12)$ \\
\hline
\end{tabular}

CI, confidence interval; ISS, Injury Severity Score (maximum 75); AIScat, Maximum Abbreviated Injury Score (maximum 6), categorized as 1 for AIS 1-2, 2 for AIS 3-4, or 3 for AIS 5-6; BP, blood pressure (systolic); GCSm, Glasgow Coma Scale, motor component (minimum 1, normally 6). 


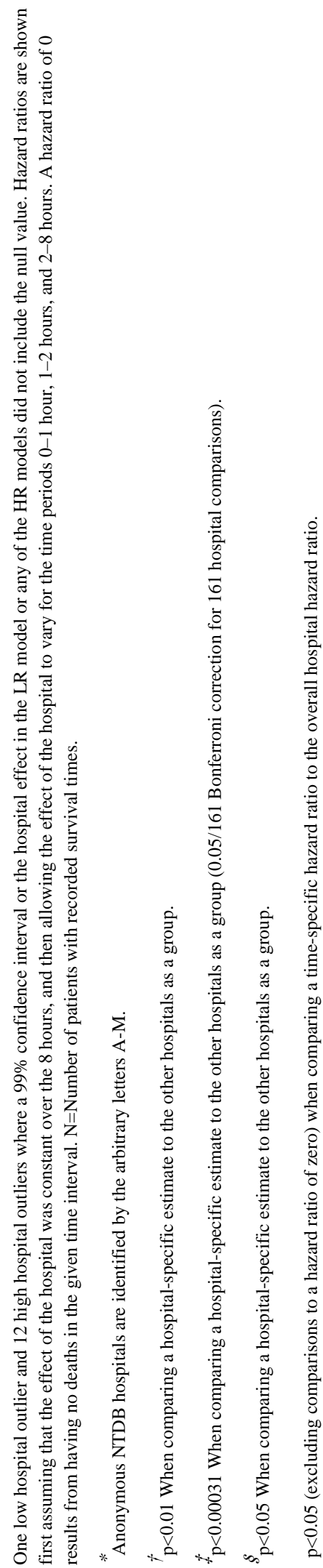

